\title{
A new target for gene therapeutics: Telomerase
}

\author{
Peter E. Nielsen
}

A new dimension has been added to "oligonucleotide-based" gene therapeutics by the paper of Norton et al.' on p.615 showing that peptide nucleic acids (PNAs) with a sequence complementary to the RNA component of human telomerase are very efficient and specific inhibitors of the enzyme in vitro.

The concept of using oligonucleotides and their analogues as gene-specific drugs based on their highly specific binding to Watson-Crick complementary (and therefore easily predictable) mRNA sequences is less than twenty years old, and led to the development of antisense gene therapeutics, which, after some growing pains ${ }^{2}$ (most probably related to the use of phosphorothioates), is now finding solid scientific ground to build on, using secondgeneration compounds. Further along the way, oligonucleotides that bind to doublestranded DNA may be developed into antigene therapeutics ${ }^{4}$, and oligonucleotides that mimic various protein-binding ligands (aptamers) ${ }^{5}$ constitute yet another application.

Human telomerase, the enzyme that ensures that telomeres (chromosome ends) are maintained in replication, is thought to be an accomplice in the collection of functions gone astray in cancer cells. Telomerase is overexpressed in the majority of human tumors, and is essential for cell immortality. Therefore the enzyme has been proposed to be a potential target for cancer therapeutics.

Telomerase carries its own telomere tem-

Peter E. Nielsen is at the department of medical biochemistry and genetics, Panum Institute,

Copenhagen, Denmark

(penpanum@biobase.dk).

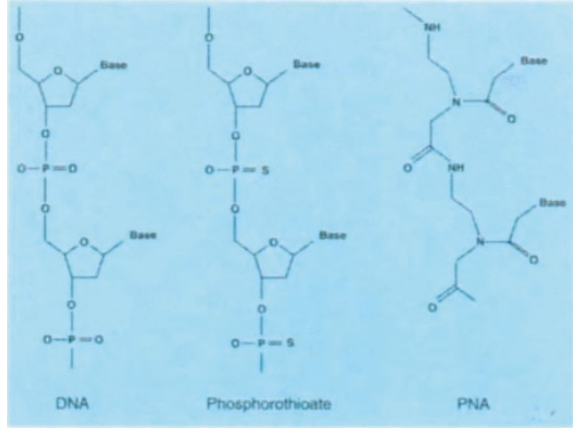

Structures of nucleotide analog oligomers.

plate in the form of an RNA molecule that is an integral part of the enzyme. Norton et al. cleverly exploited this by designing oligonucleotides that are complementary to telomerase RNA in the hope that the oligonucleotides would bind to the RNA of the enzyme and thereby function as a very specific, rationally designed, enzyme inhibitor.

They also took advantage of a recently developed DNA mimic, PNA $^{6}$, which, instead of having a negatively charged deoxyribose-phosphate backbone, is constructed with a charge-neutral, pseudo-peptide backbone consisting of $\mathrm{N}$-(2-aminoethyl)glycine units. Despite its chemical remoteness to DNA (only the nucleobases are retained), PNA has hybridization properties that are superior to those of $\mathrm{DNA}^{7}$. The ease of synthesis, along with high biological and chemical stability, has made PNA an attractive alternative to traditional DNA analogues for a large variety of applications, including gene therapeutics.

Norton et al. seem to have benefited from the uncharged nature of the PNA molecule, since they find that phosphorothioate oligonucleotides (one of the most frequently used firstgeneration DNA analogues) act as general inhibitors of telomerase, showing practically no sequence dependence.

The results have implications for both the development of gene therapeutics in general and for PNA-based drugs in particular. Human telomerase is a very interesting target for anticancer drugs, and it will, therefore, be exciting to follow the future progress of experiments in cell culture, tumor models in mice, and hopefully clinical trials. The results also show that, apart from any general superiority PNA may have over first-generation and second-generation gene therapeutics, PNAs exhibit unrivaled performance in some applications.

Naturally, it should be kept in mind that the road from demonstrating a principle of a potential drug action in vitro to actually developing a medicinally useful drug is long and full of obstacles. PNAs are only at the beginning of the road, but, as also demonstrated by the Norton paper, possible applications not attainable with traditional oligonucleotide analogues are surfacing using PNAs, and will most certainly continue to do so, owing to their unique propexties.

But the prospects for developing PNA drugs look bright.

\footnotetext{
1. Norton, J.C. et al. 1996. Nature Biotechnology 14:615-619.

Gura, T. 1995. Science 270:575-577.

3. Altmann et al. 1996. Biochemie (in press).

4élène, C. 1993. Curr. Opinion Biotechnology - 4:29.

5. Bock, L.C.et al. 1992. Nature 355:564-566.

6. Nielsen, P.E., Eghoim, M., Berg, R.H., and Buchardt, 2. 1991. Science 254:1497-1500

7. Egholm, M. et al. 1993. Nature 365:566-568.
}

\section{Breaking through gridlock on the aromatic thruway (to lower-cost specialty chemicals)}

\section{Arnold L. Demain}

Many specialty chemicals are manufactured today by chemical synthesis because costs are lower than those of biological production via the aromatic biosynthetic pathway (ARO). In

Arnold L. Demain is professor of industrial microbiology at the fermentation microbiology laboratory, room 68-223, department of biology, MIT, Cambridge, MA 02139

(demain@mit.edu). some cases, such as tryptophan, phenylalanine, and indigo, biological synthesis using microbial cells and/or enzymes are becoming competitive. In other cases, for example, para-aminobenzoic acid, folic acid, tyrosine, catechol, and quinones, production through the ARO is too expensive to compete with chemical synthesis. A major breakthrough is described on p.620 of this issue that promises major cost decreases in production of chemicals made through the ARO. This accomplishment comes from a cooperative effort of Fernando Valle, Noemí Flores, and Francisco Bolivar of the Institute of Biotechnology of Cuernavaca (Mexico), and Jimmy Xiao and Alan Berry of Genencor International in South San Francisco.

There are many obstacles on the road from external glucose to a product of the ARO. Some of the problems have already

(Cont'd on page 582) 